\title{
GRBs have preferred jet opening angles and bulk Lorentz factors
}

\author{
G. Ghisellini ${ }^{1 * \dagger}$, G. Ghirlanda ${ }^{1}$, R. Salvaterra ${ }^{2}$, L. Nava $^{3}$, D. Burlon ${ }^{4}$, G. Tagliaferri $^{1}$, S.
} Campana $^{1}$, S. Covino ${ }^{1}$, P. D'Avanzo ${ }^{1}$, A. Melandri ${ }^{1}$

1: INAF - Osservatorio Astronomico di Brera, Italy

2: INAF - IASF Milano, Italy

3: APC Université Paris Diderot, F-75205 Paris Cedex 13, France

4: Sydney Institute for Astronomy, School of Physics, The University of Sydney, NSW 2006,

Australia

\begin{abstract}
We recently found that Gamma-Ray Burst energies and luminosities, in their comoving frame, are remarkably similar. This, coupled with the clustering of energetics once corrected for the collimation factor, suggests the possibility that all bursts, in their comoving frame, have the same peak energy $E_{\mathrm{p}}^{\prime}$ (of the order of a few $\mathrm{keV}$ ) and the same energetics of the prompt emission $E_{\gamma}^{\prime}$ (of the order of $2 \times 10^{48} \mathrm{erg}$ ). The large diversity of bursts energies is then due to the different bulk Lorentz factor $\Gamma_{0}$ and jet aperture angle $\theta_{\text {jet }}$. We investigated, through a population synthesis code, what are the distributions of $\Gamma_{0}$ and $\theta_{\text {jet }}$ compatible with the observations. Both quantities must have preferred values, with log-normal best fitting distributions and $\left\langle\Gamma_{0}\right\rangle \sim 275$ and $\left\langle\theta_{\text {jet }}\right\rangle \sim 8.7^{\circ}$. Moreover, the peak values of the $\Gamma_{0}$ and $\theta_{\text {jet }}$ distributions must be related $-\theta_{\text {jet }}^{2.5} \Gamma_{0}=$ const: the narrower the jet angle, the larger the bulk Lorentz factor. We predict that $\sim 6 \%$ of the bursts that point to us should not show any jet break in their afterglow light curve since they have $\sin \theta_{\text {jet }}<1 / \Gamma_{0}$. Finally, we estimate that the local rate of GRBs is $\sim 0.3 \%$ of all local SNIb/c and $\sim 2.5 \%$ of local hypernovae, i.e. SNIb/c with broad absorption lines.
\end{abstract}

Gamma-Ray Bursts 2012 Conference-GRB2012,

May 07-11, 2012

Munich, Germany

\footnotetext{
*Speaker.

†E-mail: gabriele.ghisellini@brera.inaf.it
} 


\section{Introduction}

The spectral energy correlations in GRBs are still matter of hot debate. The isotropic equivalent energy $E_{\text {iso }}$ of the prompt phase of long GRBs correlates with the rest frame peak $E_{\mathrm{p}}$ of the $v F_{v}$ spectrum [1], [2]: $E_{\mathrm{p}} \propto E_{\text {iso }}^{0.5}$. A similar correlation (obeyed also by short events - [11]) exists between the isotropic equivalent luminosity $L_{\text {iso }}$ and $E_{\mathrm{p}}$ [24]: $E_{\mathrm{p}} \propto L_{\text {iso }}^{0.5}$.

If GRBs emit their radiation within a jet of opening angle $\theta_{\text {jet }}$, the true energy $E_{\gamma} \simeq E_{\text {iso }} \theta_{\text {jet }}^{2}$ can be estimated [7]. For $\sim 30 \mathrm{GRBs}$ with known $\theta_{\text {jet }}, E_{\gamma}$ is tightly correlated with $E_{\mathrm{p}}$ [8], [9].

The presence of outliers of the $E_{\mathrm{p}}-E_{\text {iso }}$ correlation [3], [20], [23] [5] and the presence of possible instrumental biases [4], [19], caution about the use of these correlations either for deepening into the physics of GRBs or for cosmological purposes. However, even if instrumental selection effects are present, it seems that they cannot produce the correlations we see [10] [21], [15]. Moreover, a correlation between $E_{\mathrm{p}}$ and $L_{\text {iso }}$ is present within individual GRBs as a function of time [6], [12], [13] [14].

A new piece of information recently added to the puzzle is that the energetics in the comoving frame (i.e. $E_{\text {iso }}^{\prime}, L_{\text {iso }}^{\prime}$ and $E_{\text {peak }}^{\prime}$ ) are similar for all GRBs [15]. For about 30 GRBs we [15] found that $E_{\text {iso }}\left(L_{\text {iso }}\right) \propto \Gamma_{0}^{2}$ and $E_{\mathrm{p}} \propto \Gamma_{0}$; in the comoving frame $E_{\text {iso }}^{\prime} \sim 3.5 \times 10^{51} \mathrm{erg}, L_{\text {iso }}^{\prime} \sim 5 \times 10^{48} \mathrm{erg} \mathrm{s}^{-1}$ and $E_{\mathrm{p}}^{\prime} \sim 6 \mathrm{keV}$ (see [17] for a theoretical interpretation). These results suggest that the $E_{\mathrm{p}}-E_{\text {iso }}$ and $E_{\mathrm{p}}-L_{\text {iso }}$ correlations are a sequence of different $\Gamma_{0}$ factors.

The comoving true energy $E_{\gamma}^{\prime}$ turns out to be $\sim 2 \times 10^{48} \mathrm{erg}$. In [15] we argued that to have consistency between the $E_{\mathrm{p}}-E_{\gamma}$ and the $E_{\mathrm{p}}-E_{\text {iso }}$ correlations we need $\theta_{\text {jet }}^{2} \Gamma_{0}=$ constant. The distribution of $\Gamma_{0}$ is centered around $\Gamma_{0}=65$ (130) in the case of a wind (uniform) density distribution of the circum-burst medium.

These new findings prompted us to explore the possibility that the $E_{\mathrm{p}}-E_{\gamma}$ and the $E_{\mathrm{p}}-E_{\text {iso }}$ correlations result from all bursts having the same comoving $E_{\gamma}^{\prime}$ and $E_{\mathrm{p}}^{\prime}$ but different $\Gamma_{0}$ and $\theta_{\text {jet }}$. Specifically, we [16] ask whether $\theta_{\text {jet }}$ and/or $\Gamma_{0}$ have preferential values or not, and if there is a relation between them. To this aim we have performed extensive numerical simulations, along the guidelines explained below.

\section{Simulation set up}

Fig. 1 shows the $E_{\mathrm{p}}-E_{\text {iso }}$ plane. The black points are GRBs belonging to the complete Swift sample of [22]. The large black dot corresponds to our main assumptions, i.e. all bursts, in the comoving frame, emit $E_{\gamma}^{\prime} \sim 2 \times 10^{48}$ erg at $E_{\mathrm{p}}^{\prime} \sim 1.5 \mathrm{keV}$ independent of their $\Gamma_{0}$. $E_{\mathrm{p}}^{\prime}$ is smaller $(2 \sigma)$ than the mean value derived in [15], in order to be able to reproduce GRBs lying quite close to the $E_{\mathrm{p}} \propto E_{\text {iso }}^{1 / 3}$ line. GRBs with different $\Gamma_{0}$ would lie on the $E_{\mathrm{p}} \propto E_{\gamma}$ line, giving rise to the $E_{\mathrm{p}}-E_{\gamma}$ relation. Then, by assuming a given aperture angle $\theta_{\text {jet }}$ we can calculate $E_{\text {iso }}$. The GRB will move to the right by the quantity $\left[1 /\left(1-\cos \theta_{\text {jet }}\right)\right]$ if $\theta_{\text {jet }}>1 / \Gamma_{0}$, and by the quantity $2 \Gamma_{0}^{2}$ otherwise. In the latter case, the relation between $E_{\mathrm{p}}$ and $E_{\text {iso }}$ becomes $E_{\mathrm{p}} \propto E_{\text {iso }}^{1 / 3}$. This implies that region (III) of Fig. 1 is forbidden. The other forbidden regions are region (II) because this would correspond to $\theta_{\text {jet }}>90^{\circ}$, and region (I) because we assume $1<\Gamma_{0}<8000$. All our simulated bursts will then lie on the white part of the plane. The distribution of the simulated bursts in this plane depends on the chosen distributions of $\Gamma_{0}$ and $\theta_{\text {jet }}$. We thus have a tool to find what are the best fitting distributions. 


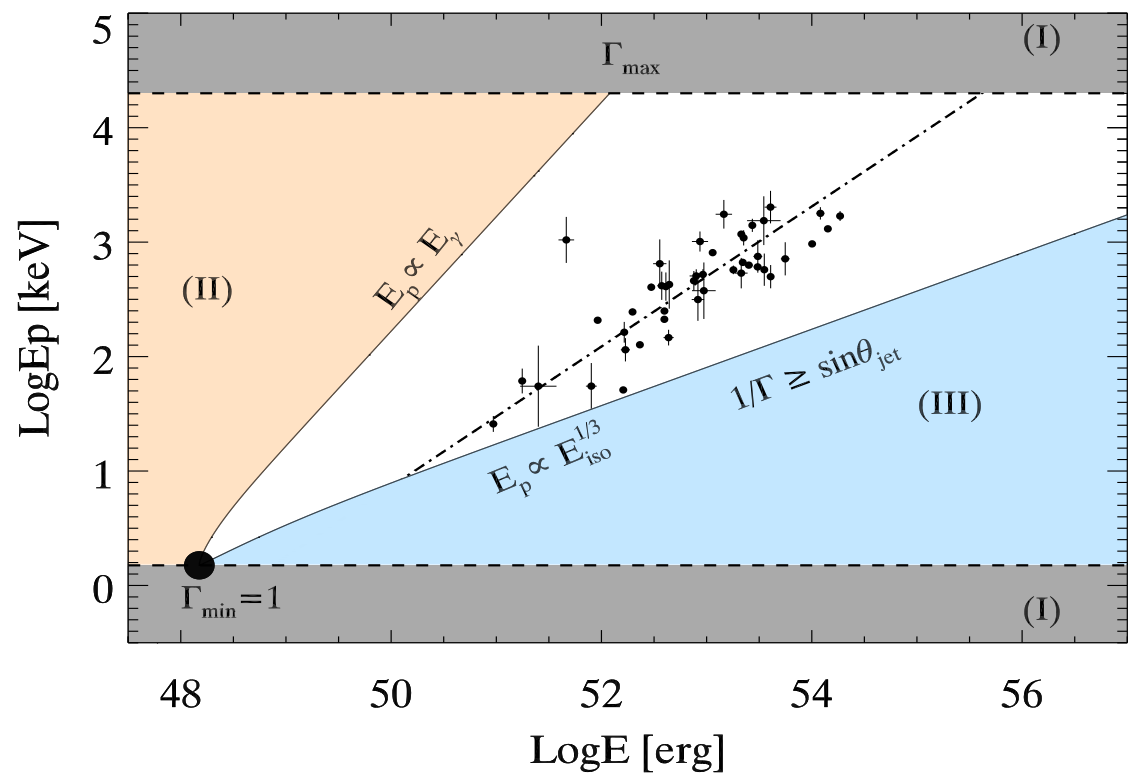

Figure 1: Rest frame plane of GRB energetics. The large black dot corresponds to all bursts having the same $E_{\mathrm{p}}^{\prime}$ and $E_{\gamma}^{\prime}$. For a given $\Gamma_{0}$, the burst moves along the line $E_{\text {peak }} \propto E_{\gamma}$. Since we assume $1<\Gamma_{0}<8000$, regions (I) are forbidden. Since all our simulated bursts have $\theta_{\text {jet }} \leq 90^{\circ}$, they cannot lie in region (II). For small $\Gamma_{0}$, the beaming cone $\sim 1 / \Gamma_{0}$ can become wider than $\theta_{\text {jet }}$. This introduces the limit $E_{\text {peak }} \propto E_{\text {iso }}^{1 / 3}$ and bursts cannot lie in region (III). Black dots correspond to the real GRBs of the Swift complete sample [22].

The steps are: i) select a redshift from the assumed redshift distribution (that is taken from [22], which includes an evolutionary term); ii) select a $\Gamma_{0}$ and calculate $E_{\mathrm{p}}$ and $E_{\gamma}$; iii) select a $\theta_{\text {jet }}$ and calculate $E_{\text {iso }}$; iv) chose a viewing angle and decide if it is pointing at us or not; v) calculate the peak flux in the appropriate band (assuming a typical Band spectrum) and decide if the burst belongs to the complete Swift sample [22] or not. Bursts in this sample have a peak flux larger than $2.6 \mathrm{ph} \mathrm{cm}^{-2} \mathrm{~s}^{-1}$, and almost $90 \%$ of them have a measured redshift. The steps are repeated until the number of simulated Swift bursts matches the real ones. Finally, we repeat 1,000 times each simulation to see how many times we can get a reasonable agreement with several observational constraints. First, we compare the simulated points of the complete Swift sample with the real ones in the $E_{\mathrm{p}}-E_{\text {iso }}$ plane. Then we compare them also in the observed planes $E_{\mathrm{p}}^{\text {obs_Fluence and }}$ $E_{\mathrm{p}}^{\mathrm{obs}}$-Peak Flux (irrespective if the redshift is known or not). Finally, we compare the distribution of simulated vs real flux and fluences of the BATSE and GBM bursts (down to limiting values that are not affected by incompleteness).

\subsection{Results}

We performed several simulations considering first that both $\Gamma_{0}$ and $\theta_{\text {jet }}$ have no preferred values, i.e. assuming that they are distributed as power-laws, changing the corresponding slopes. None of these cases is in agreement with the data. Then we assumed a broken power law either for $\Gamma_{0}$ or for $\theta_{\text {jet }}$, or for both. For the latter case we do find some agreement, but the distribution of the simulated points in the $E_{\mathrm{p}}-E_{\text {iso }}$ plane describes a linear correlation, instead of the observed 

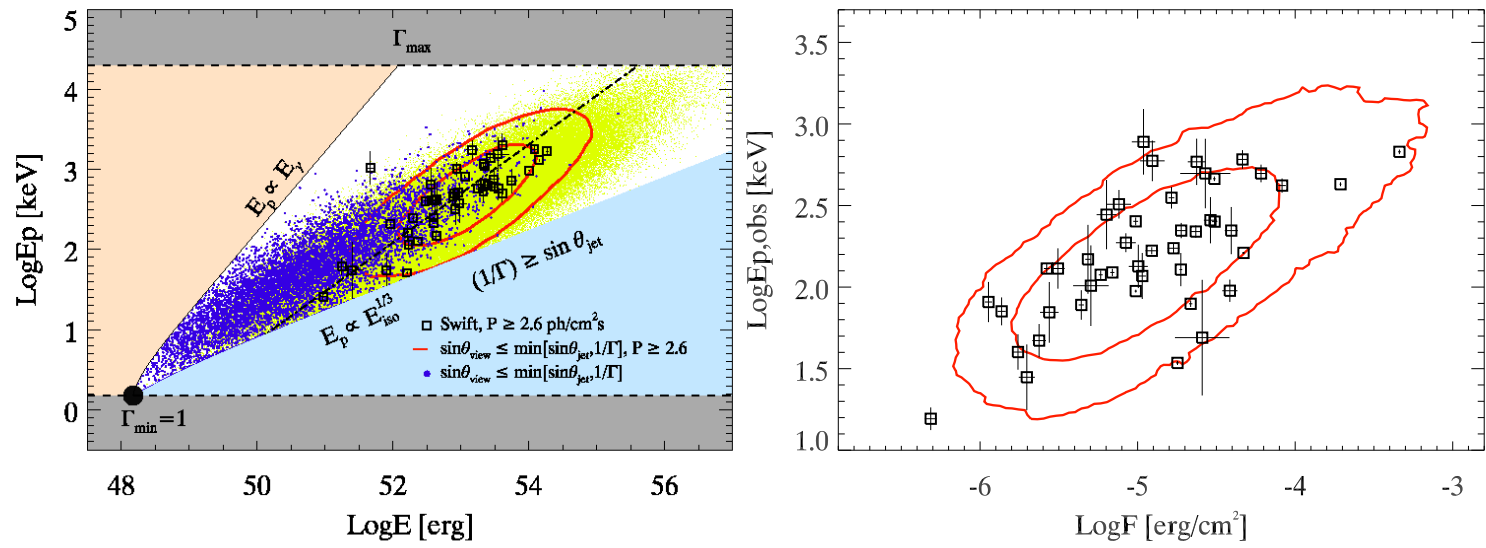

Figure 2: Simulations assuming $\log -$ normal distributions of $\theta_{\text {jet }}$ and $\Gamma_{0}$ and the relation $\theta_{\text {jet }}^{5 / 2} \Gamma_{0}=$ constant. Left panel: simulated points and real data (black) in the $E_{\mathrm{p}}-E_{\text {iso }}$ plane. Yellow points are all simulated bursts, blue points are those pointing at us, red contours are the distribution of simulated bursts $(1$ and $\sigma)$ brighter than the peak flux limit of the Swift complete sample (i.e. $2.6 \mathrm{ph} \mathrm{cm}^{-2} \mathrm{~s}^{-1}$ ). Right panel: Simulated (contours) and real points (black squares) are compared in the $E_{\mathrm{p}}^{\mathrm{obs}}$-Fluence observational plane.

\begin{tabular}{lllllll}
\hline \hline Distrib. & sample & $\sigma$ & $\mu$ & Mode & Mean & Median \\
\hline$\theta_{\text {jet }}$ & ALL & $0.916 \pm 0.001$ & $1.742 \pm 0.002$ & $2.5^{\circ}$ & $8.7^{\circ}$ & $5.7^{\circ}$ \\
& PO & $0.874 \pm 0.010$ & $3.308 \pm 0.013$ & $12.7^{\circ}$ & $40.0^{\circ}$ & $27.3^{\circ}$ \\
& PO Swift & $0.527 \pm 0.032$ & $1.410 \pm 0.043$ & $3.1^{\circ}$ & $4.7^{\circ}$ & $4.1^{\circ}$ \\
\hline$\Gamma_{0}$ & ALL & $1.475 \pm 0.002$ & $4.525 \pm 0.002$ & 11 & 274 & 92 \\
& PO & $1.452 \pm 0.020$ & $2.837 \pm 0.025$ & 2 & 49 & 17 \\
& PO Swift & $0.975 \pm 0.060$ & $5.398 \pm 0.083$ & 85 & 355 & 221 \\
\hline
\end{tabular}

Table 1: Parameter values ( $\mu$ and $\sigma$ ) obtained by fitting a log-normal function to the distributions of $\Gamma_{0}$ and $\theta_{\text {jet }}$ (Fig. 3), for all the simulated bursts (ALL), for those pointing to us (PO) and for those pointing to us and with a peak flux larger than $2.6 \mathrm{ph} \mathrm{cm}^{-2} \mathrm{~s}^{-1}$ (the flux limit of the complete Swift sample) (PO Swift). For each distribution are re ported the three moments: the mode, the mean and the median.

$E_{\mathrm{p}} \propto E_{\text {iso }}^{0.6}$. We then tried $\log$-normal distributions both for $\Gamma_{0}$ and $\theta_{\text {jet }}$. In addition we assumed that there is a relation between the average values of the two distributions. The best results are obtained with $\theta_{\text {jet }}^{5 / 2} \Gamma_{0}=$ constant (Fig. 2). Note that the slope of the $E_{\mathrm{p}}-E_{\text {iso }}$ correlation of bright bursts is harder than for faint ones (see the blue points in Fig. 2). But, curiously, these bright GRBs sample the distribution of the whole ensemble of bursts (yellow points) better than the fainter ones. This is because, if we improve our detector sensitivity, we preferentially see GRBs with larger opening angles. This makes them less energetic and enhances their probability to point at us. Fig. 3 shows (left panel) the distribution of $\Gamma_{0}$ of all simulated bursts (black), those pointing at us (blue) and those (red) that are pointing at us and have a peak flux larger than $2.6 \mathrm{ph} \mathrm{cm}^{-2} \mathrm{~s}^{-1}$ (i.e. the flux limit of the complete Swift sample). The green points correspond to the few GRBs of measured $\Gamma_{0}$ (left) or $\theta_{\text {jet }}$ (right). Tab. 1 reports the parameters of the best fitting log-normal distributions values of $\Gamma_{0}$ and $\theta_{\text {jet }}$ for all bursts (ALL), for those pointing at us (PO) and for those pointing at us with peak flux larger than $2.6 \mathrm{ph} \mathrm{cm}^{-2} \mathrm{~s}^{-1}$ (the flux limit of the complete Swift sample) (PO Swift). 

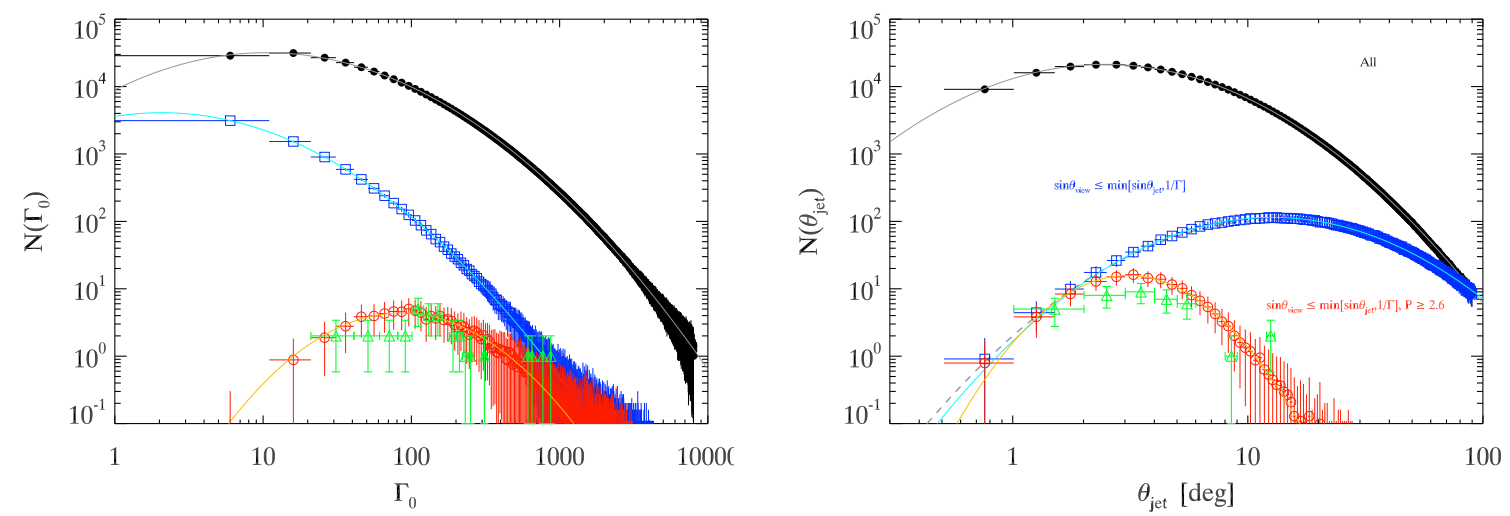

Figure 3: Distribution of $\Gamma_{0}$ (left) and of $\theta_{\text {jet }}$ (right) of GRBs. Black circles: all simulated GRBs; open blue squares: all simulated GRBs pointing at us; open red circles: GRBs pointing at us with peak flux larger than $2.6 \mathrm{ph} \mathrm{cm}^{-2} \mathrm{~s}^{-1}$ (flux limit of the complete Swift sample); green triangles: the $\sim 30$ GRBs with $\Gamma_{0}$ estimated from the onset of the afterglow [15] on the left panel, and the 27 GRBs with measured $\theta_{\text {jet }}$ collected in [8], [9] on the right panel.

\section{Conclusions}

The crucial assumption of this study is that all bursts have the same $E_{\mathrm{p}}^{\prime}=1.5 \mathrm{keV}$ and $E_{\gamma}^{\prime} \sim$ $2 \times 10^{48} \mathrm{erg}$. Although there could be a dispersion of these values, our results still hold if the width of this dispersion is not larger than the dispersion of the observed quantities. The fact that these values are independent of $\Gamma_{0}$ suggests that the dissipation mechanism giving rise to the prompt emission is not the transformation of bulk kinetic into random energy. If our assumption is true, then the $E_{\mathrm{p}}-E_{\gamma}$ relation is produced by the distribution of $\Gamma_{0}$ values, and must be linear (both $E_{\mathrm{p}}$ and $E_{\gamma}$ are proportional to $\Gamma_{0}$ ). In turn, the $E_{\mathrm{p}}-E_{\text {iso }}$ relation results from a distribution of jet aperture angles, with the caveat that, for small values of $\Gamma_{0}$, the radiation collimation angle is $1 / \Gamma_{0}$, not $\theta_{\text {jet }}$. These bursts will never have a jet-break in the light curve of their afterglow, and could be mistaken as outliers. In our simulations we find that these should be about $6 \%$ of the GRBs pointing at us. Another important outcome of our study is that we can calculate the fraction of all GRBs (whether aligned or misaligned) with respect to SN Ibc, as a function of redshift. Taking the recent estimates of the SN Ibc of [18], we find that, locally (i.e. up to $z \sim 1$ ), GRBs are $0.3 \%$ of all SN Ibc.

\section{References}

[1] L. Amati, F. Frontera, M. Tavani et al., Intrinsic spectra and energetics of BeppoSAX Gamma-Ray Bursts with known redshifts, A\&A 390 (2002) 81

[2] L. Amati, F. Frontera \& C. Guidorzi, Extremely energetic Fermi gamma-ray bursts obey spectral energy correlations, $A \& A \mathbf{5 0 8} 173$

[3] D.L. Band \& R. Preece, Testing the Gamma-Ray Burst Energy Relationships, ApJ 627 (2005) 319

[4] N.R. Butler, D. Kocevski \& J.S. Bloom, Generalized Tests for Selection Effects in Gamma-Ray Burst High-Energy Correlations, ApJ 694 (2009) 76 
[5] A.C. Collazzi, B.E. Schaefer, A. Goldstein \& R.D. Preece, A Significant Problem with Using the Amati Relation for Cosmological Purposes, ApJ 747 (2012) 39

[6] C. Firmani, J.I. Cabrera, V. Avila-Reese et al., Time-resolved spectral correlations of long-duration $\gamma$-ray bursts, MNRAS 393 (2009) 1209

[7] D.A. Frail, S.R. Kulkarni \& R. Sari, Beaming in Gamma-Ray Bursts: Evidence for a Standard Energy Reservoir, ApJ 562 (2001) L55

[8] G. Ghirlanda, G. Ghisellini \& D. Lazzati, The Collimation-corrected Gamma-Ray Burst Energies Correlate with the Peak Energy of Their $v F_{v}$ Spectrum, ApJ 616 (2004) 331

[9] G. Ghirlanda, L. Nava L., G. Ghisellini \& C. Firmani, Confirming the $\gamma$-ray burst spectral-energy correlations in the era of multiple time breaks, A\&A 466 (2007) 127

[10] G. Ghirlanda, L. Nava L., G. Ghisellini, C. Firmani \& J.I. Cabrera, The $E_{\text {peak }}-E_{\text {iso }}$ plane of long gamma-ray bursts and selection effects, MNRAS 387 (2008) 319

[11] G. Ghirlanda, L. Nava L., G. Ghisellini, A. Celotti \& C. Firmani, Short versus long gamma-ray bursts: spectra, energetics, and luminosities, A\&A 496 (2009) 585

[12] G. Ghirlanda, L. Nava L. \& G. Ghisellini, Spectral-luminosity relation within individual Fermi gamma rays bursts, 2010, A\&A, 511, 43

[13] G. Ghirlanda, G. Ghisellini \& L. Nava, Short and long gamma-ray bursts: same emission mechanism?, MNRAS 418 (2011a) L109

[14] G. Ghirlanda, G. Ghisellini \& L. Nava, Spectral evolution of Fermi/GBM short gamma-ray bursts, MNRAS 410 (2011b) L97

[15] G. Ghirlanda, L., Nava, L., G. Ghisellini et al., Gamma-ray bursts in the comoving frame, MNRAS 420 (2012a) 483

[16] G. Ghirlanda, G. Ghisellini G., R. Salvaterra et al., The faster the narrower: characteristic bulk velocities and jet opening angles of Gamma Ray Bursts, subm to MNRAS (2012b)

[17] D. Giannios, The peak energy of dissipative gamma-ray burst photospheres, MNRAS 422 (2012) 3092

[18] V. Grieco, F. Matteucci F., Meynet G. et al., Metallicity effects on the cosmic SNIb/c and GRB rates, MNRAS in press (2012) arXiv: 1204.2417

[19] D. Kocevski, On the Origin of High-energy Correlations in Gamma-Ray Bursts, ApJ 747 (2012) 146

[20] E. Nakar \& T. Piran, Outliers to the peak energy-isotropic energy relation in gamma-ray bursts, MNRAS 360 (2005) L73

[21] L. Nava, G. Ghirlanda G., Ghisellini \& C. Firmani, Peak energy of the prompt emission of long gamma-ray bursts versus their fluence and peak flux, MNRAS 391 (2008) 639

[22] R. Salvaterra, S. Campana, S.D. Vergani, et al., A Complete Sample of Bright Swift Long Gamma-Ray Bursts. I. Sample Presentation, Luminosity Function and Evolution, ApJ 749 (2012) 68

[23] A. Shahmoradi \& R.J. Nemiroff, The possible impact of gamma-ray burst detector thresholds on cosmological standard candles, MNRAS 411 (2011) 1843

[24] D. Yonetoku, T. Murakami, T. Nakamura, et al., Gamma-Ray Burst Formation Rate Inferred from the Spectral Peak Energy-Peak Luminosity Relation, ApJ 609 (2004) 935 